\title{
The first case of Granulicatella adiacens identified from a resected heart valve by next generation sequencing (NGS) in Poland
}

Anna Podgórska ${ }^{1}$, Maria Kordybach-Prokopiuk ${ }^{2}$, Maria Jaworska-Wilczyńska ${ }^{3}$, Piotr Hoffman $^{2}$, Katarzyna Biernacka ${ }^{2}$, Krzysztof Kuśmierski ${ }^{4}$, Robert Kuthan ${ }^{5}$, Tomasz Hryniewiecki $^{3}$, Anna Lutyńska ${ }^{1}$

1. Department of Medical Biology, National Institute of Cardiology

2. Department of Congenital Heart Diseases, National Institute of Cardiology

3. Department of Valvular Cardiac Defects, National Institute of Cardiology

4. Department of Cardiac Surgery and Transplantology, National Institute of Cardiology

5. Chair and Department of Medical Microbiology, Medical University of Warsaw

\begin{abstract}
In this report, we describe the course and successful treatment of complicated infective endocarditis (IE). A patient presented with a high-grade irregular fever with chills lasting at least 2 months, dyspnoea, chest pain, fatigue, weight loss, and night sweats during the previous 3 months. Above cardiac congenital disorders, he was found to have Granulicatella adiacens infective aortic valve endocarditis, presumably transmitted from the oral cavity niche. Validated metagenomic 16S rDNA next generation sequencing was used to perform taxonomic identification, allowing for specific adequate antibiotic therapy instead of empiric therapy. This paper highlights the critical role of rapid taxonomic identification of nutritionally variant streptococci crucial and the benefit of proper IE treatment to avoid relapses or fatal complications.
\end{abstract}

Introduction

Nutritionally variant streptococci (NVS) growth in standard blood culture depends on pyridoxal or cysteine supplementation. Culture-negative infective endocarditis (IE) cases sustain the main clinical problem, since NVS are slow-growing, unusual, and require additional media to be identified. In 1961, Frenkel and Hirsch described NVS as fastidious organisms that display satellite growth around colonies of other bacteria [1]. Compared to NVS, streptococci, 
enterococci, and staphylococci are easily grown, well recognized, and described as IE aetiological agents [2,3]. Based on 16S rRNA gene sequencing, Collins and Lawson [4] proposed that NVS are members of Granulicatella and Abiotrophia genera, which have rarely been demonstrated to be a specific microbiological a cause of IE. A life-threatening disease, IE is a prominent cause of morbidity and mortality, and cardiac valvular dysfunctions are known to be important risk factors in contracting IE. Dental procedures are also frequently associated with the transmission of mouth commensal bacteria, such as NVS, from the oral cavity to the blood vessels [5]. NVS are part of the healthy oral flora, intestinal tract, and urogenital system. Granulicatella adiacens are observed frequently in the oral cavity and in dental plaques, endodontic infections, and dental abscesses. IE induced by NVS is associated with high morbidity rates, frequent treatment failures, high incidence of relapses, and is a source of growing antibiotic resistance. Novel sequencing techniques may improve the identification of IE etiology when conventional tests fail to yield valid results. To the best of our knowledge, no other case of IE caused by the G. adiacens case has been reported in Poland.

\section{Keywords:}

Infective endocarditis, Granulicatela adiacens, Nutritionally variant streptococci (NVS), Nextgeneration sequencing (NGS), Aortic valve.

\section{Material and Methods}

\section{Clinical patient status}

A 20-year-old patient who had been diagnosed with a bicuspid aortic valve in childhood was admitted to the Department of Congenital Heart Diseases of the Cardinal Stefan Wyszyński Institute of Cardiology in Warsaw in July 2020 with an implanted mechanical aortic valve and suspicion of infective endocarditis (IE). The patient suffered from a high-grade, irregular fever with chills lasting at least 2 months, dyspnoea, chest pain, fatigue, weight loss, and night sweats during the previous 3 months. An echocardiographic examination performed in 2019 in another hospital revealed severe aortic regurgitation with an enlarged left ventricle (LVEDD: left ventricular end-diastolic diameter-61 mm). After transthoracic echocardiography (TTE) and computed tomography (CT) were performed, the patient was referred to the aortic valve replacement results. 


\section{Laboratory examinations}

The patient was examined for respiratory and cardiac parameters coupled with biochemical and culture microbiology testing.

\section{$\underline{\text { Valve culture }}$}

Each dissected valve sample was dissolved in $100 \mathrm{ml} \mathrm{BHI} \mathrm{(brain} \mathrm{heart} \mathrm{infusion)} \mathrm{fluid} \mathrm{medium}$ and incubated at $37^{\circ} \mathrm{C}$ with $5 \% \mathrm{CO}_{2}$ for a maximum of 10 days. Aliquots of $100 \mu \mathrm{l}$ of $\mathrm{BHI}$ cultured valve sample were removed and spread on the surface of solid microbiological media (bioMérieux, Marcy l'Etoile, France): Columbia agar with 5\% defibrinated sheep blood, MacConkey agar, bile esculin agar, mannitol salt agar, Schaedler agar with $5 \%$ defibrinated sheep blood, and chocolate agar with PolyViteXTM. For fungal culture, Sabouraud agar supplemented with gentamicin and chloramphenicol was used. All bacterial media were incubated at $37^{\circ} \mathrm{C}$ under aerobic conditions except for Schaedler medium and chocolate agar with PolyViteXTM media, which were incubated under anaerobic and microaerophilic conditions, respectively. Aerobic, anaerobic, and microaerophilic cultures were checked for growth after 24 and 48 hours. The Sabouraud medium was incubated at $30{ }^{\circ} \mathrm{C}$ under aerobic conditions for 7 days and checked every 24 hours for any growth.

\section{Blood culture}

Three sets of culture bottles, each set including a bottle for aerobic microorganisms (BD BACTEC ${ }^{\text {TM }}$ Plus Aerobic medium) and a bottle for anaerobic microorganisms (BD BACTEC ${ }^{\text {TM }}$ Lytic Anaerobic medium) were used. Each bottle was inoculated with $10 \mathrm{ml}$ of blood drawn from the patient at the bedside. Each set of blood samples were collected at 30-60 minute intervals. Samples were collected before antibiotic treatment or administration of the next antibiotic. All bottles were incubated in the BACTEC FX (Becton Dickinson) blood culture systems for 7 days after inoculation. If no bacterial growth was detected within 7 days, the blood culture was considered negative.

\section{DNA extraction}

The resected valve was collected from a patient with diagnosed IE (according to the modified Duke's criteria) in cooperation with the Department of Cardiac Surgery and Transplantology in the National Institute of Cardiology in Poland. Resected valve sections were subjected to 
culturing in the Department of Microbiology and Hospital Infections Monitoring in the National Institute of Cardiology in Poland.

The resected specimen was cut into two equal-sized pieces using sterile scissors in a biosafety cabinet. One piece of tissue was randomly selected for immediate culturing (as described above), while the other piece of tissue was snap-frozen at $-80^{\circ} \mathrm{C}$ for metagenomic sequencing and Sanger validation.

The frozen valve was thawed at room temperature for $30 \mathrm{~min}$ and cut into the smallest possible pieces with sterile scissors. Bacterial DNA was extracted from approximately $25 \mathrm{mg}$ of tissue using the Genomic Mini AX Bacteria Kit mod.7 (A\&A Biotechnology, Poland), which was modified by applying additional enzyme digestion steps with lysozyme, lysostaphin, and mutanolysin. The purity and concentration of the total bacterial DNA were determined through 260/280 $\mathrm{nm}$ absorbance measures using the NanoDropOne spectrophotometer (ThermoScientific, USA). Quantification of DNA was also performed using $1 \%$ agarose gel electrophoresis at a potential of $100 \mathrm{~V}$ (Consort EV265 Range Power Supply, Alpha Metrix Biotech, Germany). After separation, the DNA isolate was visualized under UV light after staining with an appropriate dye (Infinity VX2, Led's bar Epi-Illumination, France).

Enrichment of DNA isolated from the valve section was performed using the NEB Next Microbiome DNA Enrichment Kit (New England BioLabs, Germany). DNA was also extracted from water to ensure the absence of contaminating bacterial and reagents. In addition, DNA was extracted from the valve tissue of patients without an IE diagnosis.

\section{S rDNA next-generation Sequencing}

Paired-end sequencing libraries were constructed according to the Illumina protocol for preparing libraries. The first step was amplification of a DNA sample using specific primers to amplify a region of interest followed by attaching specific overhanging adapters. The genespecific sequences used in this protocol targeted the $16 \mathrm{~S}$ V3 and V4 region [6]. The full-length primer sequences used in the protocol to target this region were as follows: $16 \mathrm{~S}$ Amplicon PCR Forward Primer 5'-TCGTCGGCAGCGTCAGATGTGTATAAGAGACAGCCTACGGGNGGCWGC AG3'; 16S Amplicon PCR Reverse Primer 5'-GTCTCGTGGGCTCGGAGATGTGTATAAGAGAC AGGACTACHVGGGTATCTAATCC-3'. The second step involved the application of MagSi-NGS PREP Plus beads (Illumina, USA) to purify the $16 \mathrm{~S}$ V3 and V4 amplicons from free primers and primer dimers. The next step involved attaching dual indexes and Illumina sequencing 
adapters using the Nextera XT Index Kit (Illumina, USA). After the second clean-up step, the final library was quantified, normalized, and pooled. Finally, multiplexed paired-end sequencing ( $2 \times 300 \mathrm{bp}$ reads) of the $16 \mathrm{~S}$ rDNA amplicons was performed using Illumina MiSeq technology (MiSeq Reagent Kit v3 600 cycles, Illumina, USA).

High-quality sequencing data were obtained by removing low-quality reads (species with identified reads $\leq 10$ ), short reads, and duplicated reads as well as adapter contaminations. Human sequence data were mapped to a human reference genome (GRCh38.p11) using Burrows-Wheeler Alignment (BWA) software. All human sequences were removed, and the remaining sequencing data were aligned to the NCBI NT database nucleotide BASIC LOCAL Alignment Search Tool.

\section{Sanger sequencing on ribosomal DNA extracted from the heart valve}

Confirmation of NGS positive finding capillary Sanger sequencing was performed using the previously described primers targeting the 550-bp sections for the $16 \mathrm{~S}$ V3 and V4 regions. Simultaneously, the primer pair was used to amplify a 422-bp fragment unique to G. adiacens (sense: 5'-GGTTTATCCTTAGAAAGGAGGT-3' and antisense: 5'GAGCATTCGGTTGGGCACTCTAG-3') [7]. Each $20 \mu \mathrm{L}$ of the PCR reaction mixture contained 20 ng of total or microbial-enriched DNA as a template. The PCR reaction was carried out using the following protocols: an initial denaturation step was performed at $94^{\circ} \mathrm{C}$ for $10 \mathrm{~min}$, followed by 31 cycles of denaturation $\left(94^{\circ} \mathrm{C}, 30 \mathrm{~s}\right)$, annealing $\left(53^{\circ} \mathrm{C}, 1 \mathrm{~min}\right)$, and extension $\left(72^{\circ} \mathrm{C}\right.$, $1 \mathrm{~min})$, and a final elongation of $7 \mathrm{~min}$ at $72^{\circ} \mathrm{C}$.

The PCR product next underwent agarose gel electrophoresis and was visualized under UV light. Sanger sequencing was performed on a 3130xL Genetic Analyzer (Applied Biosystems, USA) for validation. Finally, the sequences obtained from Sanger sequencing were aligned to the NT database with NCBI Blast online software.

\section{Ethics}

The study protocol was approved by the hospital ethics committee (study number IK-NPIA0021-56/1643/17 at 14.11.2017). The patient provided written informed consent to participate in the study.

\section{$\underline{\text { Results }}$}

During auscultation, the patient showed a systolic-diastolic murmur. No other relevant findings were elicited. Blood tests were as follows: procalcitonin $0.08 \mathrm{ng} / \mathrm{ml}, \mathrm{CRP} 1.4 \mathrm{mg} / \mathrm{dl}$, 


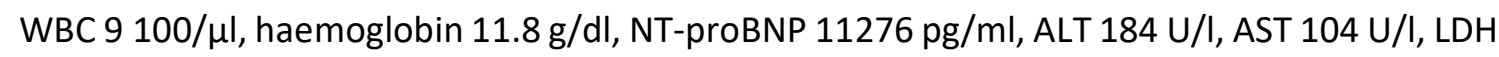
317U/I. Echocardiograms (TTE and TEE) revealed multiple vegetations and a perforation of the anterior mitral leaflet with severe mitral regurgitation. While it was difficult to evaluate the movement of the mechanical valve discs, severe paravalvular leaks with multiple vegetation were identified in the posterior part of the aortic ring. The left ventricle was enlarged (LVEDD: $70 \mathrm{~mm}$ ) with preserved systolic function (LVEF: left ventricle ejection fraction: 60\%). The right ventricle was also enlarged (RVIT: right ventricular inflow tract: $53 \mathrm{~mm}$ ) with decreased systolic function (TAPSE: tricuspid annular plane systolic excursion: $12 \mathrm{~mm}$ ). Based on these findings, a diagnosis of infective endocarditis was made. Empiric antibiotic therapy with vancomycin, gentamycin, and rifampicin was initiated. The necessity of performing cardiac surgery was confirmed by a multidisciplinary team, and the mechanical mitral valve and aortic homograft were implanted surgically. The postoperative care was complicated by multi-organ failure; as a result, arterial-venous ECMO was applied. We detected a cavity after an empty abscess in the mitral aortic curtain intraoperatively. The postoperative echocardiography examination showed normal function of the mechanical aortic valve, and a mild leakage in the posterior section of the aortic ring and moderate mitral regurgitation was found. The laboratory test indicated microcytic anemia (hemoglobin 8,6 g/dl); therefore, a gastroscopy and a colonoscopy were performed. After no signs of hemorrhage were found in the digestive tract, ferrum supplementation was prescribed. Antibiotic therapy was continued, adjusting doses appropriate to kidney function and determining the blood concentration. Parameters of inflammation as well as parameters of kidney and liver function decreased slowly and the patient's condition improved. In a control echocardiography examination, the systolic function of the borderline volume of the left ventricle (LVEDV: left ventricular end-diastolic volume: $150 \mathrm{ml}$ ) had decreased (LVEF 30-35\%). Two filiform echoes attached to the mitral subvalvular apparatus were noted. An abdominal USG showed splenomegaly without focal changes.

Experimental antibiotic therapy was applied on $26^{\text {th }}$ May; three blood samples were collected at half-hour intervals, and resected aortic and mitral valve samples were determined to be negative during 7 days of culturing. The patient received the following treatment: gentamycin i.v. ( $3 \times 0.08 \mathrm{~g}$ for 32 days and $1 \times 0.08 \mathrm{~g}$ for the following 25 days), vancomycin i.v. ( $2 \times 1 \mathrm{~g}$ for 32 days followed by a four-day break, then $1 \times 0.5 \mathrm{~g} / 2$ days during the following 21 days), and rifampicin (2x0.6 g for 29 days followed by a four-day break, then $2 \times 0.6 \mathrm{~g}$ during the following 25 days). Blood cultures collected at the end of six weeks of antibiotic therapy were still 
negative. However, during four-day breaks from vancomycin and rifampicin treatment as well as 6 months following hospitalization, the patient complained again of high-grade irregular fever $\left(39.5^{\circ} \mathrm{C}\right)$ and fatigue. To confirm the reliability of the microbiological tests, a NGS metagenomic study was implemented. Bacterial DNA was isolated from heart valve sections, a source of the entire bacteria, and underwent an NGS metagenomic procedure that was confirmed by Sanger sequencing and showed $G$. adiacens as a causative bacterium. The sequence was matched with $G$. adiacens along with 24 partial sequences of 16S RNA available in the BLAST tool library and exhibited 99\% identity with G. adiacens isolate MT459366.1. Finally, ceftriaxone with gentamycin was applied for treating IE induced by G. adiacens, resulting in a bacteriological cure.

\section{Discussion}

Infective endocarditis (IE) due to nutritionally variant streptococci (NVS) is a very rare condition and is often associated with negative blood cultures. IE caused by NVS has a higher rate of complications compared to endocarditis caused by other streptococci [8-9]. Over 100 cases of NVS infective endocarditis have been reported in the literature so far. NVS microorganisms have been found to be responsible for up to $6 \%$ of all cases of streptococcal endocarditis [10]. IE induced by NVS is characterized by frequent relapses and higher morbidity compared with infections induced with other species of streptococci. A clinical decision for rapid surgical intervention may be needed especially in penicillin-resistant cases [11]. Nevertheless, our case was culture-negative, and the surgical decision was made based on TTE and TE results clearly showing the presence of vegetations. Until metagenomic sequencing results became available, empirical therapy was initiated. After the pathogen was identified, a change in antibiotic treatment was appropriate, since previous data has showed that $G$. adiacens-infected IE patients experience a high rate of treatment success using ceftriaxone and gentamicin [9]. After changing to G. adiacens-specific targeted therapy, the patient became afebrile and in good condition symptomatically, with no relapse found on subsequent visits for follow-up during six months.

The portal of the initial entry of this taxonomic group of bacteria into the endovascular system can be related to dental procedures. These organisms are assumed to have originated from the oral cavity. Since the patient displayed poor oral hygiene and had undergone dental procedures several times before the clinical symptoms of IE developed, we can assume that 
he became infected before hospital admission. The 16S rDNA NGS analysis successfully revealed fastidious and unusual G. adiacens in the resected heart valve of the patient. About $85 \%$ of bacterial genomes contain more than 1 copy of $16 \mathrm{~S}$ rDNA; therefore, the method is able to identify bacteria genomes with high sensitivity [12]. It is possible to obtain a complete metagenomics analysis of a clinical sample in less than $30 \mathrm{~h}$ using Illumina sequencing platforms [13]. The application of NGS is of increasing clinical interest because this approach allows for faster pathogen identification compared to standard microbiological techniques. In our paper, the case described such circumstances as blood cultures and excised valve before antibiotic treatment, remained negative on day 7. The 16S rRNA gene is ubiquitous to all bacterial domains, allowing for the construction of ever-expanding sequences databases, which are critical to the unique identification of bacteria. The value of metagenomic sequencing should not be ignored, particularly in cases of culture-negative IE. Genetic analysis of microbial DNA from heart valve tissue has been included recently into the British Society for Antimicrobial Chemotherapy BSAC and ESC IE guidelines (14). Even the current version of the most authoritative and widely used Bergey's Manual of Systematic Bacteriology utilizes 16S RNA gene sequences to characterize and identify many kinds of bacteria (15). Clinicians should be very careful with reports of culture-negative results and should consider that culture-based identification is time-consuming and frequently fails to produce relevant data (since the media spectra available are restricted in many cases) within a critical time period for making rapid therapeutic decisions. Since classical symptoms of IE induced by rare and fastidious microorganisms are not typical, metagenomics studies can be the only option for clinicians to provide patients with the proper treatment necessary to overcome the fatal complications. It should be emphasized that the time required to diagnose IE using metagenomic analysis creates fast swift from empirical therapy to specific one. Since a wide spectrum of molecular tools are currently available, especially for clinically significant cases such as IE, clinicians and microbial molecular biologists should increase their level of cooperation. Close communication between clinicians and medical laboratory is necessary to connect findings with clinical pictures.

\section{Conclusions:}

Blood and valve culture-negative endocarditis is a life-threatening disease that is extremely difficult to diagnose and treat. Molecular biology techniques have higher sensitivity than 
traditional microbiological methods for detecting IE, especially when IE is related to fastidious or uncultured microorganisms (e.g. HACEK bacteria; defective streptococci - Gemella, Granulicatella, and Abiotrophia sp. - Propionibacterium acnes, Candida sp.) [16]. Our report clearly shows that there is a clinical indication to perform NGS in situations where classical microbiology methods fail. We conclude that routine high confidence identification of a microbial community at least to the genus level can be obtained for a large number of medically important organisms, especially those undescribed or fastidious, by utilizing a $16 \mathrm{~S}$ rRNA gene database generated at a particular medical health center. According to WHO, despite innovations in diagnostic tools and systems for identifying microbial infections, fast, specific, sensitive, and accurate molecular diagnostics in humans have not yet been frequently and routinely adopted. IE caused by NVS presents tremendous diagnostic and therapeutic challenges even in the modern medical era. 
1. Frenkel, A.; Hirsch, W.; Spontaneous development of L forms of streptococci requiring secretions of other bacteria or sulphydryl compounds for normal growth. Nature. 1961, 191:728-730.

2. Vandana, K.E.; Mukhopadhyay, C.; Rau, N.R.; Ajith V.; Rajath P. Native valve endocarditis and femoral embolism due to Granulicatella adiacens: a rare case report. Braz. J. Infect. Dis. 2010, 14, 634-636

3. Garibyan, V.; Shaw, D. Bivalvular endocarditis due to Granulicatella adiacens. Am. J. Case. Rep. 2013, 14: 435-438.

4. Collins,M.D.; Lawson, P.A. The genus Abiotrophia (Kawamura et al.) is not monophyletic: proposal of Granulicatella gen. nov., Granulicatella adiacens comb. nov., Granulicatella elegans comb. nov. and Granulicatella balaenopterae comb. nov. Int. J. Syst. Evol. Microbiol. 2020, 1:365-369.

5. Shailaja, T.S.; Sathiavathy, K.A.; Govindan Unni. Infective endocarditis caused by Granulicatella adiacens. Indian. Heart. J. 2013, 65: 447-449.

6. Klindworth, A.; Pruesse, E.; Schweer,T.; Peplies, J.; Quast, C; Horn, M.; Glöckner, F.O. Evaluation of general 16S ribosomal RNA gene PCR primers for classical and next-generation sequencing-based diversity studies. Nucleic. Acids. Res. 2013, 41: e1.

7. Bizzarro, M.J.; Callan, D.A.; Farrel, P.A.; Dembry, L.M.; Gallagher, P.G. Granulicatella adiacens and Early-Onset Sepsis in Neonate. Emerg.Infect.Dis. 2011, 17: $1971-1973$.

8. Giuliano, S.; Caccese, R.; Carfagna, P.; Vena, A.; Falcone, M.; Venditti, M. Endocarditis caused by nutritionally variant streptococci: a case report and literature review. Infez. Med. 2012, 20: 67-74.

9. Lin, Ch-H; Hsu, R-B. Infective endocarditis caused by nutritionally variant streptococci. Am. J. Med. Sci. 2007, 334: 235-239.

10. Padmaja, K.; Lakshmi, V.; Subramanian, S.; Neeraja, M.; Krishna, R.; Satish, O.S. Infective endocarditis due to Granulicatella adiacens: a case report and review. J. Infect. Dev. Ctries. 2014, 15: 548-550.

11. Prakash, S.S.; Rajshekar, D.; Ravindra, A.; Sneha, R.; Sastry, A.S. Acute Native Valve Endocarditis by Granulicatella adiacens. J. Clin. Diagn. Res. 2018, 12: DD01DD02. 
12. Chan, W-S.; Au, H-CH.; Chi-Ming Leung, H.; Ho, D.; Li, D.; Chan, T-L.; Lam, T-W.; Shiu-Kwan Ma, E.; Siu-Fai Tang, B. Potential utility of metagenomic sequencing for improving etiologic diagnosis of infective endocarditis. Future. Cardiol. 2019, 15: 411-424.

13. Kolb, M.; Lazarevic, V.; Emonet, S.; Calmy, A.; Girard, M.; Gaïa,N.; Charretier, Y.; Cherkaoui, A.; Keller, P.; Huber, C.; Schrenzel, J. Next-Generation Sequencing for the Diagnosis of Challenging Culture-Negative Endocarditis. Front. Med (Lausanne). 2019, 6: 203.

14. Millar, B.C.; Habib, G.; Moore, J.E. New diagnostic approaches in infective endocarditis. Heart. 2016, 15: 796-807.

15. Srinivasan, R.; Karaoz, U.; Volegova, M.; MacKichan, J.; Kato-Maeda, M.; Miller, S.; Nadarajan, R.; Brodie, E.L.; Lynch, S.V. Use of 16S rRNA gene for identification of a broad range of clinically relevant bacterial pathogens. PLoS One. 2015, 10: e0117617.

16. Tattevin, P.; Watt, G.; Revest, M.; Arvieux, C.; Fournier, P-E. Update on blood culture-negative endocarditis. Med. Mal. Infect. 2015, 45: 1-8.

Aknowledgments:

The study was supported by National Institute of Cardiology (statutory work no. 2.29/V/17 "Application of metagenomics to identify bacterial etiologies of infective endocarditis") 